\title{
Conceptual priming in a generative problem-solving task
}

\author{
RICHARD L. MARSH, MARTIN L. BINK, and JASON L. HICKS \\ University of Georgia, Athens, Georgia
}

\begin{abstract}
Three experiments explored how participants solved a very open-ended generative problem-solving task. Previous research has shown that when participants are shown examples, novel creations will tend to conform to features shared across those examples (Smith, Ward, \& Schumacher, 1993). We made the shared features of the examples conceptually related to one another. We found that when the features were related to the concept of hostility, participants' creations contained hostile features that were not part of any of the examples. These results suggest that participants will design novel entities to be consistent with emergent properties of examples shown to them. We also found that a mild hostility prime from unscrambling sentences had a similar conceptual effect. Together, the two effects suggest that conceptual priming of generative cognitive tasks will influence the cognitive aspects of the creative process.
\end{abstract}

Generative cognitive tasks, as they will be labeled here, are tasks that require participants to devise novel products. For example, participants have been asked to design novel space creatures to inhabit a distant planet (Marsh, Landau, \& Hicks, 1996; Smith, Ward, \& Schumacher, 1993; Ward, 1994; Ward \& Sifonis, 1997) or to design faces of adult and child alien creatures (Bredart, Ward, \& Marczewski, 1998). In still other studies, participants have been asked to provide novel ideas on improving their university or reducing traffic accidents (Marsh, Landau, \& Hicks, 1997). Even special populations, such as engineering students, have been asked to design novel products in their area of specialty (e.g., spill-proof mugs or bicycle racks; Jansson \& Smith, 1991). All of these studies, by definition, share the important property that the participants were cognitively engaged in generating novel entities. Although very little is known about the universal cognitive mechanisms that may support performance on such creative tasks, two general results have emerged.

First, in Ward's drawing paradigm, which requires participants to devise novel space creatures (Ward, 1994), universally participants appear to generate and to include the attributes of earth animals on their novel designs. For example, Ward found that participants' novel creations were bilaterally symmetric, had sense organs for obtaining information, and had appendages for locomotion and manipulation of the environment. One interesting aspect of these results is that features of earth animals like bilateral symmetry are rarely, if ever, given in standard attribute listing tasks in which participants explicitly write down the

This work was supported by a Sigma Xi grant-in-aid. We thank Rob Brown, Judith Johnson, Misty LeRoy, and Claire Staples for their dedicated help in collecting and scoring the data. Correspondence should be addressed to R. L. Marsh, Department of Psychology, University of Georgia, Athens, GA 30602-3013 (e-mail: marsh@meme.psy.uga.edu). features of animals (Barsalou, 1985). This conformity to features of earth animals is tenacious insofar as it persisted even when participants were asked to devise creatures "beyond their wildest imagination" (Ward, 1994, Experiment 4) and when they were asked specifically to devise creatures that were "wildly different from earth animals" (Ward \& Sifonis, 1997).

Second, when example space creatures were provided that all shared three common attributes (e.g., four legs, a tail, and antennae), participants incorporated the shared attributes into their novel designs with much greater frequency than did participants who saw no examples at all (Smith et al., 1993), and did so despite being admonished to avoid using any features of the examples (Marsh et al., 1996). The same was true of engineering students who incorporated design flaws that were present in examples shown to them (Jansson \& Smith, 1991). In terms of generative cognition, this conformity to experimenter-provided examples suggests that participants will use recently experienced information, whereas the conformity to the attributes of earth animals suggests the use of more longstanding knowledge as well.

Together, these two general findings converge on the notion that participants perform generative cognitive tasks by bringing to mind potentially relevant information about the task at hand. Because little is known concerning how participants access and subsequently use this information, this initial aspect of generative tasks remains an open and timely topic for further research. However, Perkins $(1981,1988)$ has speculated that participants attempt to recombine in unique ways whatever information is accessed in order to form an entirely new and creative product (see also Finke, Ward, \& Smith, 1992). This initial step of bringing relevant information to mind (what Perkins has labeled a harking back to old ideas) may be nothing more than bringing to mind category-relevant information. Of course, this initial step may not be as con- 
sciously explicit as Perkins's characterization contends; it could very well be a more implicit phenomenon.

Given the apparent importance of this initial step in accessing information to incorporate into novel designs, we decided to manipulate the nature of experimenterprovided examples that were shown to participants prior to their drawing of novel space creatures. Thus, we used Ward's original paradigm and Smith et al.'s (1993) variation of showing examples. The past studies that have used experimenter-provided examples obtained a very robust conformity effect despite the fact that the shared features were chosen arbitrarily. For example, creatures possessing antennae appear to be unrelated in nature to creatures having a tail. Likewise, having four legs is conceptually unrelated to whether a creature has antennae. In the only experiment that has investigated the relationship among the shared features of examples, Marsh et al. (1996, Experiment 2) found that two attributes whose cooccurrence in nature was negatively correlated (i.e., antennae and claws) resulted in a much attenuated conformity effect as compared with arbitrarily chosen features.

Given that attenuation, we predicted that the converse should also be true. If the shared features of examples are conceptually related to one another, that fact should influence overall levels of conformity. We reasoned also that any conceptual relationship among the shared attributes of examples may have important consequences for what additional features (i.e., beyond those shown in the examples) participants might incorporate into their novel designs. This prediction is made from the small, but growing, literature on conceptual combination. In that literature, individual concepts such as wooden and spoon that are placed together as a single entity, wooden spoon, often produce emergent features of the conceptual combination that are not true of the entities separately (Gagne \& Shoben, 1997; Shoben \& Gagne, 1997). One emergent property of combining wooden and spoon is that wooden spoons are large. In a similar way, the ability to talk is never listed as an attribute of pets nor of the concept of bird, but talking is nevertheless an emergent feature of the conceptual combination pet birds. The same is true of emergent features of combining rugby players with the concept of likes to knit, where participants will list bizarre as an attribute of the combination that is never listed with either concept individually (Hampton, 1997).

This work on conceptual combinations suggests that feature constellations might bring to mind (or elicit) additional attributes that features in isolation would not. In terms of generative cognition, the obvious theoretical implication is that feature constellations might elicit other features that are not part of the constellation but are nevertheless consistent with it. Some evidence in favor of this hypothesis was found when participants were instructed to design space creatures that had feathers (Ward, 1994, Experiment 2). Uniformly, the birdlike attribute of beaks was added along with the feathers. In addition, participants who were asked to draw intelligent creatures tended to draw humanoid designs that wore clothes and possessed nonclothing artifacts with much greater frequency than did participants who had not been given any information about intelligence (as reported in Ward, 1994). Although these results are not examples of emergent properties, per se, they are entirely consistent with the underlying thesis that unitary features or feature constellations can evoke other features that are consistent with one another.

On the basis of the conceptual combination literature, we predicted that a set of conceptually related features that were shared across examples might elicit (as an emergent property) additional features that were also consistent with the concept. That is, showing participants examples with three shared features that converged on a particular concept might cause participants to incorporate additional, but concept-related, features into their novel designs. Thus, our manipulations concern conceptually related features that are shared across examples, and our prediction is that additional concept-related features might emerge in participants' novel drawings.

We chose the concept of hostility as the conceptual attribute to emerge from three shared features. Hostility was chosen because it was easily defined in terms of attributes to be included on experimenter-provided examples (e.g., fangs, spikes, and weapons). Likewise, hostility is easily defined in terms of additional attributes that participants might generate that were consistent with it (e.g., horns, claws, etc.). Hostility was also chosen because it has a history of being easily induced in laboratory participants (Srull \& Wyer, 1979, 1980). We were unsure whether showing participants three slightly hostile creatures might alter their conception of space creatures or affect their cognitive processing more generally. Although we state clearly at the outset that it might be impossible to disambiguate among these alternatives, choosing a concept that could be induced in more than one fashion would at least allow us to collect empirical data that would begin to address this issue. Using tried-and-true manipulations also alleviated our need to assess whether participants were placed in a slightly hostile mood by more general priming manipulations.

In Experiment 1, we showed participants three creatures that shared three hostile attributes. Performance in that condition was compared with that in a control group that saw no examples. In Experiment 2, we tested the generality of the results from Experiment 1 by orthogonally crossing whether participants saw hostile examples with whether they received a general hostility prime not specifically tied to the experimenter-provided examples. On the one hand, perhaps only hostility tied to the examples would cause participants to include other features consistent with concept of hostility. On the other hand, perhaps generative cognitive tasks are sensitive to more global forms of conceptual priming. As described later, two different control conditions were tested. In Experiment 3 , we attempted to eliminate the effect that conceptual hostility priming had in Experiment 2. 


\section{EXPERIMENT 1}

Our goal in conducting Experiment 1 was to ascertain whether three shared features that converged on a single concept (i.e., hostility) would cause concept-related features to be incorporated into participants' novel designs. Smith et al. (1993) and Marsh et al. (1996) have shown the standard conformity effect - that participants will incorporate shared features from examples into novel designs more frequently than participants who are not shown any examples. However, we also predicted that participants might incorporate additional features that are consistent with the hostility concept, but that were not shown on the examples, into their designs.

\section{Method}

Participants. Forty-four University of Georgia undergraduates participated in exchange for partial course credit. Participants were tested in small groups of 5-7 participants. These groups were assigned randomly to one of two experimental conditions, as defined shortly. For all experiments, cell sizes for the experimental conditions are displayed in the first column of Table 1 .

Materials and Design. The basic design consisted of two groups of participants. The examples group viewed three experimenterprovided space creatures as examples prior to drawing their own novel space creatures, whereas the control group did not view anything. For the examples group, three space creatures were designed that were similar to those used by Smith et al. (1993) and Marsh et al. (1996). As in those earlier articles, the three example creatures all shared three critical features. In this experiment, those features were fangs, spikes, and weapons. We chose these features because we believed that together they formed a coherent core representing the concept of hostility. The specific instantiation of each shared feature differed perceptually on each of the three examples, and especially so for the feature of weapon (e.g., gun, club, etc.). Each example was drawn from both a front and a side view and its features were labeled. To be consistent with the previous studies, an innocuous caption was added to each example. This caption was entirely unrelated to the concept of hostility. For the drawing task, participants were given prepared booklets containing opaque brown paper that prevented them from reviewing or revisiting their earlier cre- ations. The example drawings were projected on a screen at the front of the room, as described next.

Procedure. We followed the procedure used by Marsh et al. (1996) except that the participants in this experiment engaged in an unrelated experiment prior to being tested in this one. This experiment followed the unrelated one in order to equate the time spent in the laboratory with the conditions tested in Experiments 2 and 3 (i.e., for experimental control and consistency). At the start of this experiment, the following orienting instructions were read:

Imagine a planet just like earth existing somewhere else in the universe. It is currently uninhabited. Your task is to design new creatures to inhabit the planet. Within the 20 minutes allotted, draw as many new and different creatures of your own creative design as you are able. Duplication of creatures now extinct or living on the planet earth is not permitted.

These were the identical orienting instructions that we have used previously. Participants in the examples group then viewed three creatures. These were displayed for $30 \mathrm{sec}$ each. After the viewing period, participants were admonished not to copy any aspect of the examples with the following instructions, taken from Marsh et al. (1996):

The examples we just showed you were created by participants in another group that we tested earlier. We showed you these examples in order to help you think about creating your own original creatures and to get your creative juices flowing. However, we do not want you to copy any aspect of the examples. Please concentrate on creating your own original creations.

Participants in the control condition did not see examples, but they were asked to sit quietly for $90 \mathrm{sec}$ before drawing to equate the time spent by participants in the examples condition. Participants in both conditions then drew creatures of their own novel design for $20 \mathrm{~min}$. The drawing booklets reminded participants to provide both front and side views of each creature, to label each part of it, and to provide a one- or two-sentence description of it. After completing one creature, participants continued to the next drawing page in their booklet to design another new creature, and so forth, throughout the 20 -min drawing period.

\section{Results and Discussion}

The creatures were scored independently by two raters who were unaware of the objectives of the experiment. Each was instructed to score each creature for every fea-

Table 1

Output Metrics of Creativity and Metrics of Conformity for Experiments 1-3

\begin{tabular}{|c|c|c|c|c|c|c|c|c|c|c|}
\hline \multirow[b]{2}{*}{$N$} & \multirow[b]{2}{*}{ Sentences } & \multirow[b]{2}{*}{ Examples } & \multicolumn{2}{|c|}{$\begin{array}{c}\text { Number of } \\
\text { Creatures }\end{array}$} & \multicolumn{2}{|c|}{$\begin{array}{c}\text { Number of } \\
\text { Features }\end{array}$} & \multicolumn{2}{|c|}{$\begin{array}{c}\text { Target } \\
\text { Conformity }\end{array}$} & \multicolumn{2}{|c|}{$\begin{array}{l}\text { Conceptual } \\
\text { Conformity }\end{array}$} \\
\hline & & & Avg. & $S E$ & Avg. & $S E$ & Avg. & $S E$ & Avg. & $S E$ \\
\hline \multicolumn{11}{|c|}{ Experiment 1} \\
\hline 21 & None & None & 4.29 & .35 & 6.69 & .70 & 0.01 & .01 & 0.03 & .02 \\
\hline 23 & None & Hostile & 3.70 & .18 & 7.93 & .59 & 0.09 & .02 & 0.12 & .03 \\
\hline \multicolumn{11}{|c|}{ Experiment 2} \\
\hline 34 & Neutral & None & 3.94 & .24 & 6.53 & .35 & 0.01 & .01 & 0.05 & .02 \\
\hline 32 & Neutral & Hostile & 3.93 & .25 & 6.75 & .54 & 0.08 & .02 & 0.18 & .03 \\
\hline 31 & Hostile & None & 3.64 & .17 & 7.29 & .34 & 0.09 & .02 & 0.18 & .04 \\
\hline 32 & Hostile & Hostile & 3.43 & .18 & 6.84 & .60 & 0.08 & .02 & 0.15 & .03 \\
\hline 32 & Syllable & None & 3.88 & .20 & 6.49 & .32 & 0.02 & .01 & 0.04 & .01 \\
\hline 33 & Syllable & Hostile & 3.63 & .13 & 6.02 & .29 & 0.08 & .02 & 0.17 & .03 \\
\hline \multicolumn{11}{|c|}{ Experiment 3} \\
\hline 23 & Hostile & Neutral & 3.96 & .22 & 5.91 & .41 & 0.02 & .01 & 0.04 & .02 \\
\hline 22 & Hostile & Hostile & 3.95 & .19 & 6.62 & .49 & 0.07 & .02 & 0.13 & .04 \\
\hline
\end{tabular}


ture that it contained, regardless of whether the person had explicitly labeled it or not. As in our earlier work, features could be identified with little ambiguity and disputes were settled in conference between the two raters and the second author. The results reported are those based on the postconference agreement of the raters and the second author.

Unless otherwise noted, statistical significance by chance does not exceed $5 \%$. The first two rows of Table 1 summarize the results. In this experiment and those that follow, two metrics of creative output and two metrics of conformity are reported. Each will be considered in turn. In terms of creative output, both the control and the examples groups produced approximately four novel drawings $[t(42)=1.51, S E D=.38$, n.s.]. Another metric of creative output is the elaborateness of participants' creations. In the past, we have used the average number of features as an estimate of how elaborate the creatures were. Although participants who saw examples included approximately one more feature on average than participants assigned to the control condition, this difference was not statistically significant $[t(42)=1.64, S E D=.89$, n.s. $]$. These null results indicate that the provision of examples does not change output metrics of creativity.

In contrast, large differences in conformity were observed. Target conformity was calculated in the following way (see also Smith et al., 1993). Each creature was assigned 1 point if it contained the features of fangs, spikes, or weapons (for a maximum of 3 points). This score was divided by 3 to obtain a proportion. This procedure was followed for each of the drawings produced by a given participant, and the proportions were averaged over creatures to arrive at a single proportion for each participant. The averages across participants assigned to the two conditions are given in Table 1. Thus we are calling the three shared features of the examples target conformity instead of the earlier term, conformity.

The second metric of conformity we have labeled conceptual conformity, as displayed in the final columns of Table 1 . This metric was calculated in the same way as target conformity except that a different set of features was used. In analyzing the master coding lists used by Marsh et al. (1996), we were able to identify three additional features that could be construed unambiguously as consistent with the concept of hostility. These features were horns, claws, and protective coverings (e.g., armor, shells, plates, etc.). Thus, the conceptual conformity metric included only these three additional features.

We analyzed the data with a $2 \times 2$ mixed analysis of variance (ANOVA) model with the between-subjects factor of condition and the within-subjects factor of type of conformity. In that analysis, both the effect of condition $\left[F(1,42)=8.52, M S_{\mathrm{e}}=.02\right]$ and the type of conformity $\left[F(1,42)=5.58, M S_{\mathrm{e}}=.01\right]$ were statistically significant but the interaction was not. As is evident from Table 1, participants shown the examples displayed larger conformity effects, and the conceptual conformity effect was larger than the target conformity effect. Thus, partic- ipants who saw the experimenter-provided examples included the shared features of fangs, spikes, and weapons much more frequently than did participants who did not see any examples [simple effect: $t(42)=2.8, S E D=.02$ ] This result replicates both Marsh et al. (1996) and Smith et al. (1993), and it can be viewed as a constraint on creativity because it supports the counterintuitive notion that providing examples does not always facilitate participants' performance.

However, as is also evident from Table 1, participants who saw examples that contained a core of three hostile features included additional hostile features with much greater frequency than did participants who saw no examples at all [simple effect: $t(42)=2.04, S E D=.08$ ]. The conceptual conformity score demonstrates that when a set of common features embodies a concept such as hostility across a set of examples, people will generate on their own and then use additional features that are consistent with that concept. Therefore, when asked to generate something novel, participants are sensitive not only to specific features they have encountered before but also to emergent properties from feature constellations. This effect could represent an implicit learning phenomenon in the following sense. As participants study the experimenter-provided examples, the core features of fangs, spikes, and weapons may converge on, or otherwise evoke, the concept of hostility. From the set of three features, the notion that space creatures are hostile may be an emergent property that is implicitly learned from the examples. The emergent property of hostility is expressed in the generative task when participants use features other than those included in the examples.

Our only reason for considering these effects as implicit comes from postexperimental questionnaires in which we specifically asked participants what they noticed, if anything, about the three experimenter-provided examples. No one in the present experiment, and only 4 participants out of 142 who saw the hostile examples in Experiments $1-3$, mentioned the concept of hostility and was able to report even one of the common features (in one case, that all three had a weapon). Rather, participants often listed that all the creatures had eyes or arms, or some other feature (which was not always correct). Against what baseline these few participants should be statistically compared is unclear. However, we do know that universally, participants failed to report to us any explicit knowledge of our manipulation, and therefore their conformity is unlikely to reflect any conscious strategy concerning hostility. Thus, we believe that the conceptual conformity score demonstrates that creative problem-solving tasks are not immune to conceptual priming effects. In addition, to the extent that the target conformity score represents a constraint on creativity, the conceptual conformity score captures people's attempts to express a concept (in this case, hostility) in novel ways as well. Thus, participants' novel use of horns, claws, and protective coverings could be construed as creative attempts to conform to an 
implicit belief that space creatures might be hostile. Because conceptual conformity was found to be greater than target conformity, together these metrics suggest that participants' creative expressions can outweigh the constraints of providing examples.

\section{EXPERIMENT 2}

Showing participants a set of three examples that shared a core set of three hostile features greatly increased their propensity to incorporate an entirely different set of hostile features into their designs. That effect is novel and interesting because it extends Heit's (1992) finding that participants can detect and reliably use very small correlations among features. Presumably, the shared features of the examples could be correlated (i.e., co-occur in nature) with the additional features that participants independently generated and used on their novel designs.

We wondered, however, whether the introduction of the concept of hostility had to be part and parcel of the examples or whether it could be introduced in an "unrelated" task. On the one hand, participants may generate and use these additional features that appear to be consistent with the features that they saw on the experimenterprovided examples. By this account, participants include other hostile features perhaps as a consequence of holding the belief that space creatures are hostile. We make no claims about whether such a belief would be implicitly or explicitly held, although the data from the postexperimental questionnaires in Experiments 1-3 would suggest the former. On the other hand, the concept of hostility may be primed more independently: Viewing hostile space creatures may merely activate the concept of hostility (i.e., independent of any beliefs about the hostility of space creatures). Once activated, the hostile concept itself affects generative cognition in a way that resulted in the differences observed in Experiment 1.

As we stated at the outset, disambiguating between these alternatives in a completely convincing manner might be difficult, and it is not necessarily our goal to do so in this article. We can, however, assess whether introducing the concept of hostility in an unrelated task (similar to providing examples) prior to the drawing task affects generative cognitive performance. Our approach was to introduce a mild hostility prime approximately $12 \mathrm{~min}$ prior to drawing. The manipulation that we used was taken from the social literature (Srull \& Wyer, 1979). In that study, unscrambling 60 mildly hostile sentences in an "unrelated" experiment led participants to interpret socially ambiguous behaviors as indicative of negative personality traits. The general manipulation works with a variety of traits, such as happy and sad, as well as mean (Higgins, Rholes, \& Jones, 1977; Wyer \& Srull, 1986). Moreover, the more negative terms one is exposed to, the greater the effect on dispositional inferences (e.g., Bargh \& Pietromonoaco, 1982, found a linear relationship).
If hostility as a concept affects this generative task independent of being part of the examples, then participants who unscramble hostile sentences may design creatures that reflect the primed concept of hostility. The target and conceptual conformity scores used in Experiment 1 would reflect that propensity. If hostility as a concept must be integrally part of the examples, then unscrambling hostile sentences should not increase the probability of generating and using hostile features in the design of novel creatures. In Experiment 2, the unscrambling of either neutral or hostile sentences was orthogonally crossed in a between-subjects design with whether or not participants saw hostile examples. Although these four conditions constituted our basic design, we added two additional conditions for experimental control. In these conditions, participants were exposed to the hostile scrambled sentences, but they merely counted the number of syllables in the words. Thus, they were exposed to the identical set of words, but not to the conceptual result of hostility that comes from unscrambling the sentences.

\section{Method}

Participants. One hundred ninety-four undergraduates volunteered to participate in exchange for partial credit toward a research requirement. None had participated in Experiment 1. Testing was conducted in small groups of approximately 6 participants.

Materials and Design. The three space creatures used in Experiment 1 were used again. Recall that they all shared the three common features of spikes, fangs, and weapons. For the examplesindependent manipulation of hostility, scrambled sentences that had either a hostile valence or a neutral valence were taken from Watson, Pritzker, and Madison (1955). The neutrally valenced sentences were used for experimental control in terms of exposure to sheer amount of information and to equate the lengths of the experimental sessions across tasks and conditions. When unscrambled, all sentences were four words long. Participants who unscrambled hostile sentences ended up writing down things such as "Smash the rusty lock" and "Go straight to hell." In contrast, the unscrambled versions of the neutral sentences generally mentioned the same or more neutrally valenced concepts such as "Fix the old lock" and "Please go to sleep." The unscrambled solution was unambiguous, and, of course, none of the concepts mentioned in the sentences were related to the six features that constituted the target or conceptual conformity scores.

Procedure. In Experiment 1, the two groups tested could be easily labeled. In the present experiment, it was more difficult to label the conditions mnemonically. Because the sentence manipulation always preceded viewing experimenter-prov ided examples (when they were viewed), we capitalized on this feature to refer to the basic four conditions of interest, plus two additional conditions conducted for experimental control. Unscrambling neutral sentences was either followed by not viewing any examples (neutral-none condition) or was followed by viewing three examples that shared the three hostile features (neutral-hostile condition) (see Table 1). These two conditions basically replicated the design of Experiment 1 except that in this experiment, participants first unscrambled neutrally valenced sentences. In a similar fashion, unscrambling hostile sentences was either followed by not viewing any examples (hostile-none condition) or followed by viewing three examples that shared the three hostile features (hostile-hostile condition). Thus, the first word in a condition label refers to the valence 
of sentence manipulation and the second word refers to whether examples were seen.

For experimental control, we tested two additional conditions in which participants were exposed to the hostile scrambled sentences but were not required to unscramble them. Rather, they were asked to count the number of syllables in each word and to write down the total of the four words combined. This syllable-counting task controlled for exposure to the same basic information, and also allowed us to avoid exposing participants to the concept of hostility that emanates from the actual unscrambling. Therefore, in the syllable-none condition, participants counted the number of syllables in the hostile scrambled sentences and did not see experimenter-provided examples, whereas in the syllable-hostile condition, they counted syllables and were then shown examples that shared three hostile features.

Participants were told that they would be taking part in several unrelated experiments in order to receive credit for a full research hour. Participants first unscrambled 60 sentences that were either hostile or neutral, depending on their assigned condition, or counted the number of syllables in words constituting the scrambled hostile sentences. The scrambled sentences (e.g., "Lock the rusty smash") were shown four at a time projected from a $35-\mathrm{mm}$ slide projector. The slides were displayed for 1 min each, which was ample time for participants to write the four unscrambled sentences down on numbered response sheets, or to write the number of syllables. Thus, the sentence-priming task required a total of $15 \mathrm{~min}$. Following that priming, participants rated famous and fictitious proper names for how famous they believed the person to be. This fame-judgment task required $12 \mathrm{~min}$ and served as a filler task between the sentencepriming manipulation and the drawing task. After the filler task participants either viewed three example space creatures or sat quietly under the same instructions and procedure as those used in Experiment 1 (see Table 1 for a synopsis of the design). All participants then drew novel space creatures for $20 \mathrm{~min}$.

\section{Results and Discussion}

As in Experiment 1, two unaware raters scored the designs. As summarized by the first four rows under $\mathrm{Ex}$ periment 2 in Table 1 , a $2 \times 2 \times 2$ mixed ANOVA model was conducted, with the three factors being the valence of unscrambled sentences, whether or not examples were seen, and the type of conformity. We will return to the syllable-counting conditions later. For the output metrics of creativity, the numbers of creatures drawn did not vary with whether or not examples were seen $[F(1,125)<1.0]$. However, there was a statistically marginal effect suggesting that participants who unscrambled neutral sentences drew on average one third of a creature more than those who unscrambled hostile sentences $[F(1,125)=$ $3.42, p=.07]$. There were no other significant effects. For the complexity metric of the average number of features, none of the main effects or interactions were statistically significant $[$ all $F \mathrm{~s}(1,125)<1]$. Therefore, there was not much evidence that the manipulations produced changes in overall output. As discussed next, however, these same manipulations changed the specific features that constituted participants' creations.

The conformity scores (as defined in Experiment 1) are shown in the last two sets of columns in Table 1. As is evident there, both target and conceptual conformity scores were much smaller in the neutral-none condition than in the remaining three conditions, in which equiva- lent performance was observed (still momentarily excluding the two syllable conditions). Recall that each of these three latter conditions contained some sort of hostility prime. Thus, these data suggest that either type of manipulation of hostility - the one tied specifically to the examples or the one that was independent of the examplesincreased participants' propensity to include hostile features on their novel creations. The interesting aspect of these data is that the hostile sentence priming caused participants to generate and to use the same set of six hostile features as those used by participants who were explicitly shown examples that contained only three of these features. The statistical analyses showed that providing the sentential prime increased conformity $[F(1,125)=$ $\left.4.21, M S_{\mathrm{e}}=.03\right]$, as did providing examples $[F(1,125)=$ $\left.3.91, M S_{\mathrm{e}}=.03\right]$. Obviously, these factors interacted, because the neutral-none condition differed from the three remaining conditions $\left[F(1,125)=3.90, M S_{\mathrm{e}}=.03\right]$. An important result replicating the findings from Experiment 1 was that conceptual conformity was greater than target conformity $\left[F(1,125)=37.73, M S_{\mathrm{e}}=.01\right]$.

Having described the main conditions of interest, we turn now to our syllable-counting control conditions. In order to demonstrate the absence of differences, we compared the results of these two conditions with those of the two conditions in which neutrally valenced sentences were unscrambled. A $2 \times 2 \times 2$ ANOVA with the factors of task (unscrambling neutral sentences vs. counting syllables), examples (provided vs. not provided), and type of conformity (target vs. conceptual) indicated that the task variable was not significant $[F(1,127)<1.0]$. The provision of hostile examples increased the number of hostile features by either conformity metric, as reported earlier $[F(1,127)=16.10]$ and caused conceptual conformity to be greater than target conformity $[F(1,127)=$ 19.42]. None of the interactions were statistically significant [all $F \mathrm{~s}(1,127)<1.0]$. Thus, these results reinforce the notion that the hostility priming is a function of the unscrambling and generation of hostile sentences, and not merely a function of exposure to the components of the sentences.

As in Experiment 1, providing examples increased participants' propensity to use the common, shared features of the examples, despite experimenter instructions to avoid doing so. As measured in the conceptual conformity score, however, introducing the concept of hostility across the shared features, thereby making it an integral part of the examples, also induced participants to include additional, unmentioned features that were consistent with the concept of hostility. This finding replicated the results of Experiment 1. Perhaps the most interesting result of Experiment 2 was finding that the introduction of the hostility concept need not be integrally bound to the examples. Participants who unscrambled mildly hostile sentences 12 min prior to drawing nevertheless included more hostile features in their novel creations than did participants in the control conditions. Importantly, neither conformity score was 
larger for the hostile-hostile condition, which included both hostility manipulations, than for the hostile-none and the neutral-hostile conditions, which included only one hostility manipulation. Thus, the two priming manipulations were not additive, suggesting that they may have affected cognitive processing in a similar, if not identical, manner. Because both the hostile examples and the hostile sentence-priming manipulations elicited the production of hostile features, these results suggest that generative cognitive tasks such as devising space creatures are sensitive to conceptual priming. Moreover, conceptual priming can be introduced by exposure to material that is either related or unrelated to the generative task at hand. In the General Discussion section, we consider why unrelated tasks might influence generative cognitive tasks in this way.

\section{EXPERIMENT 3}

Given the fairly robust effects of the two hostility manipulations on participants' drawings, our goal in Experiment 3 was to ascertain whether we could inoculate, or otherwise remove, the effects of the hostile sentence priming. We reasoned that the strongest inoculation would come from showing participants neutral experimenterprovided examples that had no emergent properties at all. Therefore, we tested a hostile-neutral condition in which participants unscrambled the hostile sentences, performed an unrelated distractor activity, and then saw neutral space creatures whose features collectively did not evoke any particular concepts. Performance in this condition was compared with that in the hostile-hostile condition of Experiment 2 . The latter condition was chosen because the hostile-hostile condition elicited conformity scores that were equivalent to both the hostile-none and the neutralhostile conditions of Experiment 2. Using it provided us with the opportunity to verify once again that the simultaneous use of both manipulations of hostility does not have any additive or synergistic effect.

\section{Method}

Forty-five participants were recruited from the same population as that used in Experiments 1 and 2. They were tested in small groups, with assignment to the two experimental conditions randomly determined on the basis of their arrival at the laboratory. The 60 hostile sentences used in Experiment 2 were used again. They were presented to participants in both conditions in an identical fashion to that described before. Following the fame-judgment distractor period, participants in the hostile-hostile condition viewed three example space creatures that shared the common features of fangs, spikes, and weapons for $30 \mathrm{sec}$ each. In contrast, the participants in the hostile-neutral condition viewed three creatures that did not share conceptually related common features and had no hostile features (but that were equated to the hostile examples in terms of the overall number of features). The groups were otherwise identical, with all participants admonished not to copy any features of the examples before they drew creatures of their own novel design for $20 \mathrm{~min}$ (see Experiment 1 for the details).

\section{Results and Discussion}

Participants' novel creations were scored in an identical manner to that reported for Experiments 1 and 2. The results are summarized in the last two rows of Table 1. By output metrics of creativity, the groups did not differ from each other in terms of the total number of creatures drawn $[t(43)<1.0, S E D=.29$, n.s.] or in the average number of features that constituted their novel creations $[t(43)<$ $1.0, S E D=.63$, n.s.]. The two groups did differ, however, in their expressions of the concept of hostility during the drawing task. Target conformity to the features of fangs, spikes, and weapons was higher in the hostile-hostile condition than in the hostile-neutral condition $[t(43)=2.01$, $S E D=.03, p=.05]$. Similarly, conceptual conformity to the additional hostile features of horns, claws, and protective covering was greater in the hostile-hostile condition than in the hostile-neutral condition $[t(43)=2.50, S E D=$ $.06]$. These simple effects translated into a main effect of greater conformity in the hostile-hostile condition $[F(1,43)$ $\left.=6.26, M S_{\mathrm{e}}=.02\right]$ and a main effect of greater conceptual conformity than target conformity $[F(1,43)=4.53$, $\left.M S_{\mathrm{e}}=.02\right]$. The interaction was not significant.

These results suggest that seeing neutral examples following a conceptual hostility priming manipulation reduces participants' propensity to include hostile features on their novel designs. Thus, more recent exposure to neutral material, especially in conjunction with examples that participants might view as relevant to the generative task, can eliminate the conceptual priming effects of earlier exposure. Although the generality of these effects will need to be tested with concepts other than hostility, and with generative tasks other than drawing novel space creatures, performance in the hostile-hostile condition again showed no additive or synergistic effects of applying both priming manipulations as compared with the results from Experiment 2 . We now consider more generally how these experiments are informative about the cognitive processing in generative problem-solving tasks.

\section{GENERAL DISCUSSION}

The novel and important results from these experiments can be summarized as follows. When examples that share conceptually related features are shown just prior to participants' generating novel exemplars, participants not only use those shared features, but they also generate and use additional features that are consistent with the emergent underlying concept from the primed feature constellation. More specifically, when three examples that shared three hostile features were shown, participants used not only those three features, but also additional hostile features that had not been experimentally introduced prior to the drawing task. Even more subtlely, the hostile features do not even need to be shown, as was demonstrated in the conditions in which participants unscrambled mildly hostile sentences. An examination of Table 1 should im- 
press the reader that these effects are remarkably robust. We interpret the sentential manipulation associated with hostility as a conceptual form of priming that affected how participants approached this particular generative cognitive task. Only after the introduction of neutral examples was the sentential priming effect greatly reduced.

Our contribution to this new literature on generative cognition must be viewed largely as empirical at this point. Theoretically, these experiments do not specify the exact mechanisms that have given rise to the conceptual conformity results, which are arguably the most important results reported in this article. Although speculative, one possibility is that participants shown examples might implicitly (or more explicitly) possess the belief that space creatures are more hostile entities due to the presence of the core features. Another possibility is that the constellation of hostile features might evoke a more hostile mindset, which then acts to increase the number of hostile features that participants use in designing their creatures. We have not collected empirical evidence that would allow us to specifically determine whether conceptual priming or a hostile mind-set is the genesis of the conceptual conformity displayed by participants. Clearly, however, participants were unable to explicitly tell us that the examples were hostile in response to our specific query of whether they noticed any regularities in the examples. Moreover, the finding that the "unrelated" task of unscrambling mildly hostile sentences yielded conceptual conformity even in the absence of experimenter-provided examples speaks to the possibility that participants' mind-sets at the time they undertake a generative cognitive task will influence performance. If this is the case, these experiments clearly demonstrate an implicit effect on creative cognition.

We believe that it is important that having participants both unscramble sentences and see examples that possessed hostile features yielded no additive or synergistic effect. In addition, providing neutral examples eliminated the effects of the hostile sentential manipulation. In both cases, we believe that seeing the examples just prior to the drawing task is likely to have eliminated any effect of having done the unscrambling. That is, once participants are given information via examples, that information may become the implicit standard by which to compare their own performance on the task. Thus, the inoculation effect of the neutral examples and the lack of any greater impact on conformity from receiving both hostility manipulations suggests that seeing examples sets boundary limits on the sorts of features that are incorporated into novel space creatures.

Our results are consistent with other findings on the cognitive consequences of providing participants with additional information about a task. For example, Jacoby and Whitehouse (1989) presented participants with a recognition memory test. In an unaware condition, just prior to a word's appearance a subthreshold prime of the word or a different word was presented. Performance in this condition was compared with that in an aware condition in which the prime was presented supraliminally.
When the prime and target matched in the unaware condition, participants responded in favor of the word more often than when they were aware of the prime. In a similar fashion, Whittlesea, Jacoby, and Girard (1990) presented items for a recognition test with either $20 \%$ or $40 \%$ visual white noise. When participants were unaware that the visual noise level was being manipulated, more items under the $20 \%$ masking were called "old" because they were perceived more easily. In contrast, when participants were made aware of this fact, performance in the two masking conditions was equated. From results such as these, it becomes readily apparent that providing participants with additional information through examples could very easily change how the the generative task is approached.

The interesting aspect about the conformity to the examples is that participants were explicitly told to avoid using the features on the examples. They did, however, incorporate them more often than controls, who saw no examples. Although this result might reflect some sort of unconscious plagiarism (see Brown \& Murphy, 1989; Marsh \& Bower, 1993) or a basic constraint on creativity, the data contain other evidence that participants were genuinely attempting to be creative and to avoid the features of the examples they had seen. For the conditions in which hostile examples were presented, the conceptual conformity score is essentially a metric of the expression of hostility in ways other than the features that were present on the examples. The statistical analyses consistently demonstrated that conceptual conformity was greater than target conformity. Thus, we believe that participants were attempting to avoid the features of the examples and creatively chose additional hostile features that had never been mentioned in the experiment at all.

We freely admit that the hostile sentential manipulation could have led to the conformity effects we observed by an entirely different set of cognitive mechanisms. The parsimonious explanation, however, is that both manipulations activated the notion of hostility in a way that increased participants' propensity to think of and to incorporate hostile features in their novel space creatures. The important finding, revealed especially by the results of the unscrambling of hostile sentences by itself (i.e., the hostile-none condition of Experiment 2), is that realworld mind-sets are likely to find their way into creative designs. The twists and turns of a novel; the inclusion of features in a painting; and the content and form of lyrics, melodies, and poems are all likely to be influenced by the mind-sets of their creators. Bower (1986; see also Bower, 1996) has argued that mind-sets of therapists can alter the diagnoses that they make of their patients. Although the rendering of a diagnosis might rarely be considered creative behavior, we argue that it is something of a puzzle to fit together the various symptoms of a patient in order to render an informed and conclusive judgment.

Obviously, we did not find evidence that mind-set and specific examples interacted in any way; but we tested only one frame of mind-hostility. As the study of gener- 
ative cognition moves beyond its infancy, perhaps people's mind-set and examples of existing designs can be shown in the laboratory to produce interesting interactions. Those interactions are likely to be informative as to just how people might be biased as designers of novel products. Although those interactions may be found in domains other than the drawing task used here, the results in Table 1 provide an important point of departure for investigating a host of other variables that are likely to influence generative cognition in equally reliable ways.

\section{REFERENCES}

Bargh, J., \& Pietromonoaco, P. (1982). Automatic information processing and social perception: The influence of trait information presented outside of conscious awareness on impression formation. Journal of Personality \& Social Psychology, 43, 437-449.

BARSALOU, L. W. (1985). Ideals, central tendency, and frequency of instantiation as determinants of graded structure in categories. Journal of Experimental Psychology: Learning, Memory, \& Cognition, 11, 629-654.

Bower, G. H. (1986). Prime time in cognitive psychology. In P. Eelen (Ed.), Behavior therapy: Beyond the conditioning paradigm (pp. 2247). Amsterdam: North-Holland.

BOWER, G. H. (1996). Reactivating a reactivation theory of implicit memory. Consciousness \& Cognition, 5, 27-72.

BRedart, S., WARD, T. B., \& MARCZEWSKI, P. (1998). Structured imagination of novel creatures' faces. American Journal of Psychology, 111, 607-625.

Brown, A. S., \& MurPhy, D. R. (1989). Cryptomnesia: Delineating inadvertent plagiarism. Journal of Experimental Psychology: Learning, Memory, \& Cognition, 15, 432-442.

FINKE, R. A., WARD, T. B., \& SMITH, S. M. (1992). Creative cognition: Theory, research, and applications. Cambridge, MA: MIT Press.

GAGNe, C., \& Shoben, E. J. (1997). The influence of thematic relations on the comprehension of nonpredicating combinations. Journal of Experimental Psychology: Learning, Memory, \& Cognition, 23, 71-87.

HAMPTON, J. A. (1997). Emergent attributes in combined concepts. In T. B. Ward, S. M. Smith, \& J. Vaid (Eds.), Creative thought: An investigation of conceptual structures and processes (pp. 83-110). Washington, DC: American Psychological Association.

HEIT, E. (1992). Categorization using chains of examples. Cognitive Psychology, 24, 341-380.

Higgins, E. T., Rholes, W. S., \& Jones, C. R. (1977). Category accessibility and impression formation. Journal of Experimental Social Psychology, 13, 141-154.

JACOBY, L. L., \& Whitehouse, K. (1989). An illusion of memory: False recognition influenced by unconscious perception. Journal of $E x$ perimental Psychology: General, 118, 126-135.
JANSSON, D. G., \& SMith, S. M. (1991). Design fixation. Design Studies, 12, 3-11.

MARSH, R. L., \& BowER, G. H. (1993). Eliciting cryptomnesia: Unconscious plagiarism in a puzzle task. Journal of Experimental Psychology: Learning, Memory, \& Cognition, 19, 673-688.

Marsh, R. L., Landau, J. D., \& Hicks, J. L. (1996). How examples may (and may not) constrain creativity. Memory \& Cognition, 24, 669-680.

Marsh, R. L., LANDAU, J. D., \& Hicks, J. L. (1997). The contribution of inadequate source monitoring to unconscious plagiarism during idea generation. Journal of Experimental Psychology: Learning, Memory, \& Cognition, 23, 886-897.

Perkins, D. N. (1981). The mind's best work. Cambridge, MA: Harvard University Press.

PERKINS, D. N. (1988). Creativity and the quest for a mechanism. In R. J. Sternberg \& E. E. Smith (Eds.), The psychology of human thought (pp. 310-336). New York: Cambridge University Press.

SHOBEN, E. J., \& GAGNe, C. (1997). Thematic relations and the creation of combined concepts. In T. B. Ward, S. M. Smith, \& J. Vaid (Eds.), Creative thought: An investigation of conceptual structures and processes (pp. 31-50). Washington, DC: American Psychological Association.

Smith, S. M., WARd, T. B., \& Schumacher, J. S. (1993). Constraining effects of examples in a creative generation task. Memory \& Cognition, 21, 837-845.

SRULL, T. K., \& WYER, R. S. (1979). The role of category accessibility in the interpretation of information about persons: Some determinants and implications. Journal of Personality \& Social Psychology, 37, 1660-1672.

SRULL, T. K., \& WYER, R. S. (1980). Category accessibility and social perception: Some implications for the study of person memory and interpersonal judgments. Journal of Personality \& Social Psychology, 38, $841-856$.

WARD, T. B. (1994). Structured imagination: The role of category structure in exemplar generation. Cognitive Psychology, 27, 1-40.

WARD, T, B., \& SIFONIS, C. (1997). Task demands and generative thinking: What changes and what remains the same? Journal of Creative Behavior, 31, 245-259.

Watson, R. E., PritzKer, L., \& MAdison, P. (1955). Hostility in neurotics and normals. Journal of Abnormal \& Social Psychology, 50, 36-41.

WhitTlesea, B. W. A., JACOBY, L. L., \& Girard, K. (1990). Illusions of immediate memory: Evidence of an attributional bias for feelings of familiarity and perceptual quality. Journal of Memory \& Language, 29, 716-732.

WYER, R. S., \& SRULL, T. K. (1986). Human cognition in its social context. Psychological Review, 93, 322-359.

(Manuscript received June 11, 1997; revision accepted for publication January $21,1998$. ) 